\title{
Photosensitizer Nanoparticles Boost Photodynamic Therapy for Pancreatic Cancer Treatment
}

Cite as

Nano-Micro Lett.

(2021) 13:35

Huanyu Yang ${ }^{1}$, Renfa Liu $^{2}$, Yunxue $\mathrm{Xu}^{2}$, Linxue Qian $^{1}{ }^{凶}$, Zhifei Dai $^{2} \bowtie$

Received: 29 August 2020

Accepted: 31 October 2020

Published online: 4 January 2021

(C) The Author(s) 2020

\section{HIGHLIGHTS}

- Current clinical studies of photodynamic therapy against pancreatic cancer are reviewed.

- Advantages of nanoparticles in boosting therapeutic efficacy of photodynamic therapy for pancreatic cancer treatment are summarized.

- Challenges and outlook for the future development of nanoparticles-based photodynamic therapy in human are discussed.

\begin{abstract}
Patients with pancreatic cancer (PCa) have a poor prognosis apart from the few suitable for surgery. Photodynamic therapy (PDT) is a minimally invasive treatment modality whose efficacy and safety in treating unresectable localized PCa have been corroborated in clinic. Yet, it suffers from certain limitations during clinical exploitation, including insufficient photosensitizers (PSs) delivery, tumor-oxygenation dependency, and treatment escape of aggressive tumors. To overcome these obstacles, an increasing number of researchers are currently on a quest to develop photosensitizer nanoparticles (NPs) by the use of a variety of nanocarrier systems to improve cellular uptake and biodistribution of photosensitizers. Encapsulation of PSs with NPs endows them significantly higher accumulation within PCa tumors due to the increased solubility and stability in blood circulation. A num-
\end{abstract}

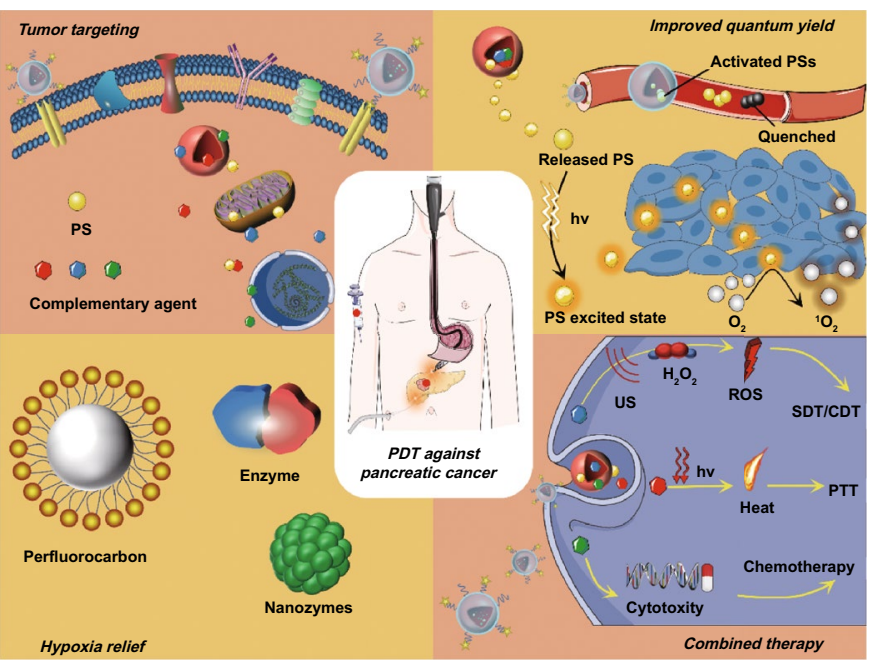
ber of approaches have been explored to produce NPs co-delivering multi-agents affording PDT-based synergistic therapies for improved response rates and durability of response after treatment. This review provides an overview of available data regarding the design, methodology, and oncological outcome of the innovative NPs-based PDT of PCa.

KEYWORDS Photodynamic therapy; Photosensitizer; Nanoparticle; Pancreatic cancer; Combined therapy

Huanyu Yang, Renfa Liu and Yunxue Xu have contributed equally to this work.

$\bowtie$ Linxue Qian, qianlinxue2002@163.com; Zhifei Dai, zhifei.dai@pku.edu.cn

1 Department of Ultrasound, Beijing Friendship Hospital, Capital Medical University, No. 95 Yongan Road, Xicheng District, Beijing 100050, People's Republic of China

2 Department of Biomedical Engineering, College of Engineering, Peking University, No. 5 Yiheyuan Road, Haidian District, Beijing 100871, People's Republic of China 


\section{Introduction}

Pancreatic cancer (PCa) remains a lethal disease for which 5 -year survival rate of all diagnostic stages combined is only about 9\% [1]. For localized resectable tumors, surgery followed by adjuvant chemotherapy (gemcitabine plus capecitabine) is the standard of care, and the 5-year survival rate in these patients is about 30\% [2]. However, more than $80 \%$ of patients have locally advanced or metastatic disease at diagnosis and are unsuitable for curative surgical resection [3]. Chemotherapy is the main therapeutic option for inoperable patients. However, $\mathrm{PCa}$ is relatively chemotherapyrefractory, even with the most aggressive FOLFIRTNOX regimen (folinic acid, 5-fluorouracil, irinotecan, and oxaliplatin), the median overall survival of patients with metastatic disease does not exceed one year (11.1 months), at the cost of cumulative systemic toxicity [4].

Photodynamic therapy (PDT) has emerged as a potential alternative therapy for unresectable patients due to its effectiveness against chemo- and radio-resistant cells [5, 6]. PDT relies on the accumulation of photosensitizer (PS) in tumors, which upon light irradiation transfers absorbed photon energy or excited electrons to surrounding oxygen to generate singlet oxygen $\left({ }^{1} \mathrm{O}_{2}\right)$ and other forms of reactive oxygen species (ROS) [7, 8]. The generated ROS can directly trigger intrinsic apoptotic pathways associated with oxidative damage to mitochondria [9-11]. Thus, PDT can directly activate the late stages of the apoptotic program that bypasses many cell death signaling pathways.

PDT has been used as part of the palliative care for PCa and exhibited potential superiority in existing clinical trials. Due to the special position and vital function of pancreas, the pancreatic cancer surgery is the most difficult to conduct and operative mortality varied between 0 and $10 \%$ [12]. On the contrary, PDT can be conducted in a minimally invasive way by inserting optical fiber into the tumor to deliver light. Since the PSs can be selectively targeted to tumors, the potential damage to vital tissues can be minimized. However, it suffers from certain limitations during clinical exploitation, including insufficient PSs delivery, tumor-oxygenation dependency, and treatment escape of aggressive tumors [13]. Recently, PDT-related treatments mediated by nanoparticles (NPs) have been proven by basic researches to be able to overcome these obstacles [14]. NPs-based strategy is attractive for PDT of PCa. On the one hand, the encapsulation of
PSs in NPs could improve the efficacy of PDT by improving the solubility and stability of PSs, increasing targeted delivery of PSs in tumors, and relieving the tumor hypoxia by oxygen supplying. On the other hand, NPs can also be used to load other therapeutic agents for PDT-based synergistic therapy [5]. The present review analyzed the principal application strategies, mechanism of action, and available experimental data of NPs-mediated PDT against PCa.

\section{Clinical Status of PDT for Pancreatic Cancer}

PDT has been reported to be effective and safe for the management of nonmetastatic locally advanced pancreatic cancer in several pilot phase I/II studies, where laser light was delivered along the optical fibers positioned percutaneously through the anterior abdominal wall or via transgastric/ transduodenal puncture under endoscopic ultrasound (EUS) guidance, to overcome the limitations of light attenuation in penetrating biological tissues. The first clinical study of PDT for PCa was conducted by Bown et al. in 16 patients, using mTHPC (meta-Tetra(hydroxyphenyl)chlorin), and the fiber was inserted percutaneously using a combination of ultrasound imaging and computed tomography (CT) guidance [12]. Substantial tumor necrosis was detected with CT following PDT treatment and all the patients except two left hospital in 10 days. In a further phase I/II clinical study, mTHPC was replaced with verteporfin (liposomal benzoporphyrin derivative) for its reduced skin photosensitivity and longer wavelength absorption peak with deeper tissues penetration [15]. Due to the proximity of the endoscope to the pancreas, optical fiber can be inserted into the tumor via a transgastric or transduodenal approach under EUS guidance. Choi et al. described the first application of EUSguided PDT (EUS-PDT) using chlorin e6 (Ce6) derivative in four patients with locally advanced pancreaticobiliary cancer and demonstrated the technique feasibility of EUS-PDT [16]. In another phase I study of EUS-PDT using porfimer sodium, 6 of $12(50 \%)$ patients showed increased pancreatic tumor necrosis after EUS-PDT [17]. These studies confirm that PDT can achieve controllable tumor necrosis with low adverse event profile, although precautions should be taken for tumors invading the duodenal wall or gastroduodenal artery. It is of value for locally advanced disease in local tumor control and combined application before or after 
chemoradiotherapy. Besides, some locally advanced patients can become qualified for surgery after PDT due to tumor downstaging $[15,17]$.

However, PDT still suffers from certain limitations in these early phase studies. On the one hand, PDT against PCa is not completely free from severe adverse events such as gastrointestinal hemorrhage and duodenal obstruction, partly due to the poor selectivity of the existing PSs [7]. This nonselective distribution of PSs induces the risk of photodamage to the adjacent organs, and inefficient PSs accumulation in tumor sites. On the other hand, during follow-up after PDT, both liver metastases and tumor regrowth around edges of treated area occurred in some cases [12, 15]. The underlying reason may be related to the insufficient ${ }^{1} \mathrm{O}_{2}$ yield within tumor. Conventional PSs such as porphyrins and other tetrapyrrole derivatives have poor water solubility and are prone to aggregate in physiological solutions through $\pi-\pi$ stacking and hydrophobic interaction $[7,18]$. This aggregation can severely impair ${ }^{1} \mathrm{O}_{2}$ yield via aggregation caused quenching (ACQ) effect [19]. Moreover, single-modal PDT is not enough to cure $\mathrm{PCa}$ and combination with other therapy such as chemotherapy is critical to prolong the survival rate of Pca [20-22]. Thus, various kinds of PS-loaded nanoparticles were developed in the aim of overcoming these limitations (Fig. 1).

\section{Nanoparticles-Mediated PDT for Pancreatic Cancer}

Encapsulating PSs in NPs can improve the solubility and stability of PSs, avoid self-quenching, and thereby increase ${ }^{1} \mathrm{O}_{2}$ yield [23-25]. In addition, NPs can also be designed to deliver oxygen or generate oxygen in situ, thereby relieving tumor hypoxia, which is detrimental for efficient PDT [26-28]. The NPs can inherently target to tumors through the enhanced permeability and retention (EPR) effect, a unique phenomenon of solid tumors including PCa related to their anatomical and pathophysiological differences from normal tissues [29]. Moreover, the NPs can also be modified with specific ligands to achieve active tumor targeting [30].

\subsection{Improving Solubility of PSs and ${ }^{1} \mathrm{O}_{2}$ Yield}

Most PSs such as porphyrins and other tetrapyrrole derivatives are hydrophobic and contain big heteroaromatic rings, which make PSs prone to aggregate by $\pi-\pi$ stacking and hydrophobic interaction [7, 18]. By rational design of the PS encapsulation, PSs can be loaded with very high loading content while avoiding self-quenching [31-33]. Liang et al. fabricated a series of PS-loaded nanoparticles based on porphyrin-grafted lipid (PGL) [32, 34, 35]. The porphyrin molecules can be loaded in the PGL nanoparticles with a drug loading as high as $38.45 \%$ [32]. The orderly arranging mode of porphyrins and alkyl chains in the PGL molecules prevents PSs from aggregating and self-quenching, even at a very high density of PSs. Compared with the free porphyrin molecules, the generation of ${ }^{1} \mathrm{O}_{2}$ by PGL nanoparticles in aqueous solution was elevated by several fold. Ding et al. developed a polyphosphoester-based nanocarrier (NP-PPE) to deliver Ce6 for PDT of PCa [36]. NP-PPE/ Ce6 was prepared by self-assembly of amphiphilic diblock copolymer of methoxypolyethylene glycols (mPEG) and polyphosphoester, denoted as mPEG-b-PHEP, and Ce6 was loaded in the hydrophobic polyphosphoester core (Fig. 2). NP-PPE/Ce6 kept Ce6 in encapsulated state during circulation, but rapidly released $\mathrm{Ce} 6$ in the acid endosome or lysosome within cancer cells due to decreased hydrophobicity of polyphosphoester core. The ability of ${ }^{1} \mathrm{O}_{2}$ generation of NP-PPE/Ce6 or free $\mathrm{Ce} 6$ was assessed by fluorescence intensity of the oxidized product dichlorofluorescein (DCF). As expected, NP-PPE dramatically improved accumulation of $\mathrm{Ce} 6$ and ${ }^{1} \mathrm{O}_{2}$ generation in tumor relative to that of free Ce6 molecules, and NP-PPE/Ce6-mediated PDT treatment showed an enhanced antitumor efficacy on human BxPC-3 PCa-bearing mice.

\subsection{Oxygen Supplying}

Oxygen is a key requirement for ${ }^{1} \mathrm{O}_{2}$ generation in PDT and the efficacy of PDT can be compromised by hypoxic tumor microenvironment (TME). An important application of NPs is to establish an oxygen self-sufficient PDT nanoplatform for treating hypoxic $\mathrm{PCa}$, which is characterized by a dense desmoplastic stroma as well as hypovascular and hypoperfused tumor vessels [37]. Oxygen can be loaded in NPs using oxygen-absorbing materials, such as liquid perfluorocarbon [38]. On the other hand, various in situ oxygen production nanoparticles, partially relying on the overproduced $\mathrm{H}_{2} \mathrm{O}_{2}$ in TME, are conceived as an alternative method to improve tumor oxygenation during PDT [28, 39]. 


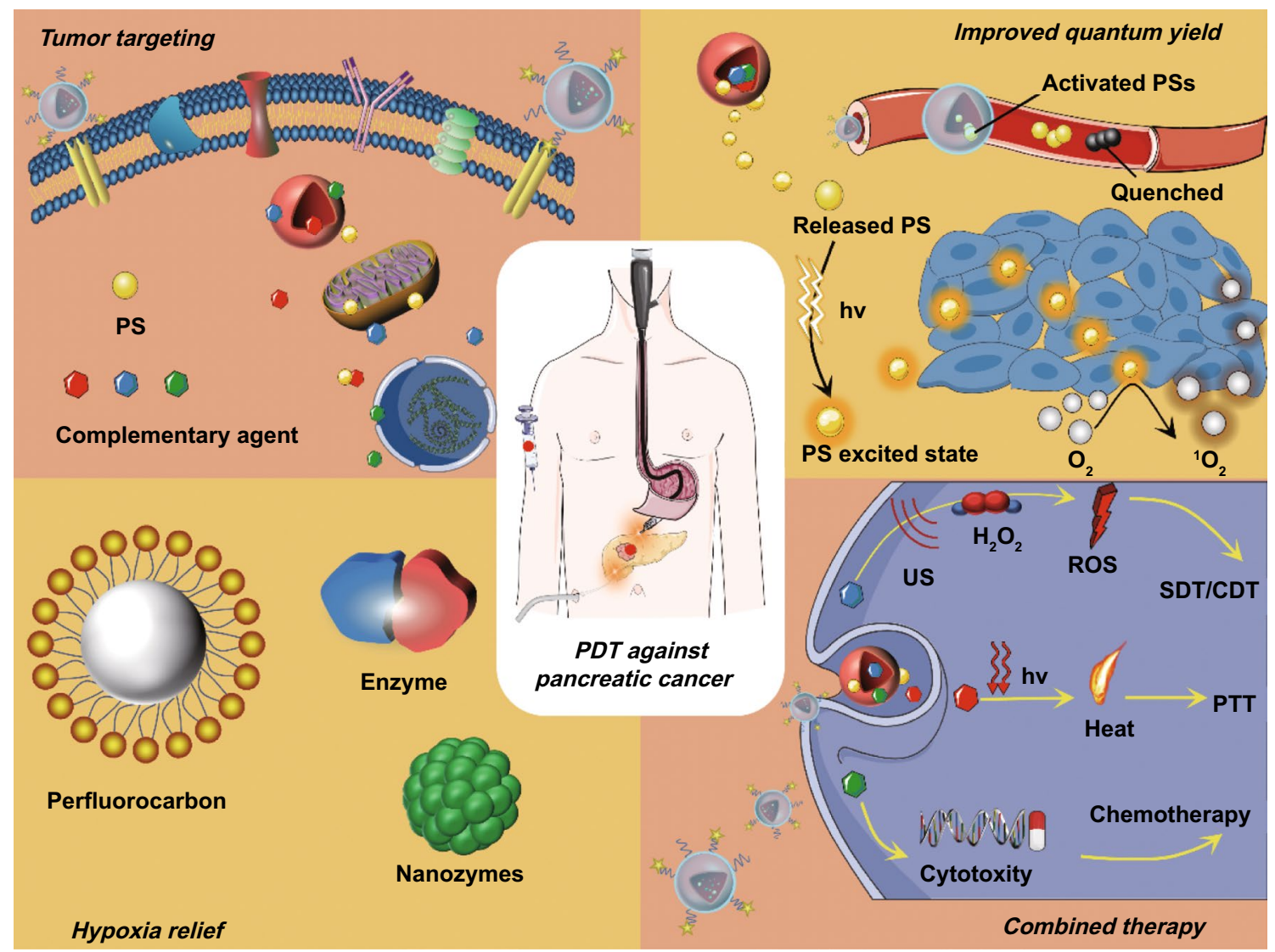

Fig. 1 Schematic illustration of PS-loaded NPs for localized photodynamic destruction of PCa

A recent study by $\mathrm{Hu}$ et al. is a good example of NPsbased oxygen self-sufficient PDT platform, which is fabricated by loading catalase and methylene blue (MB) in mesoporous hierarchical zeolite nanocarriers (Fig. 3) [40]. Catalase loaded in the zeolite could efficiently relieve tumor hypoxia by continuously decomposing endogenic $\mathrm{H}_{2} \mathrm{O}_{2}$ and in situ producing a large amount of $\mathrm{O}_{2}$ inside tumor, thereby promoting efficacy of $\mathrm{O}_{2}$-dependent PDT. As shown by $2 \mathrm{D}$ photoacoustic imaging for oxygenated hemoglobin $(\lambda=850 \mathrm{~nm})$, after the intratumoral injection of the zeolitecatalase-MB (ZCM) nanocapsule, the blood oxyhemoglobin level in tumor tissues increased over time and reached its maximum after 3-h post-injection. Moreover, the zeolite nanocarrier can be degraded under acid conditions, exhibiting high biocompatibility and biodegradability. In addition to natural catalase, nanozymes, synthetic nanomaterials with inherent enzyme-like characteristics, can also be designed with a range of enzymatic activity and have been explored as potential solutions to ameliorate tumor hypoxia during PDT.
Kang et al. prepared a hollow Ru-Te nanorod (RuTeNR) with inherent oxidase, peroxidase, superoxide dismutase (SOD), and catalase-type activity, which also exhibited photothermal and photodynamic combinatorial effect under near-infrared (NIR) laser irradiation [41]. This RuTeNR was synthesized through solvothermal galvanic replacement of the sacrificial Te nanorod template with $\mathrm{Ru}(\mathrm{III})$ as the replacement metal cation. By the peroxidase-like activity, RuTeNRs decomposed $\mathrm{H}_{2} \mathrm{O}_{2}$ in TME into hydroxy radicals (HO) exerting cytotoxic effect. Meanwhile, RuTeNRs converted $\mathrm{H}_{2} \mathrm{O}_{2}$ into $\mathrm{O}_{2}$ by their catalase-like activity to promote ${ }^{1} \mathrm{O}_{2}$ generation of PDT. Under photoactivation, RuTeNRsmediated PDT effect synchronized with their photothermal effect through segregated photonic pathways in suppressing MIA PaCa-2 pancreatic tumors.

In another significative study by $\mathrm{Li}$ et al. [42], the tumor hypoxia alleviation strategy relied on photothermal effect to decompose $\mathrm{H}_{2} \mathrm{O}_{2}$ into $\mathrm{O}_{2}$. In detail, Ce6/cypate-conjugated poly(amidoamine) dendrimers (CC-PAMAM) and $\mathrm{H}_{2} \mathrm{O}_{2}$ 

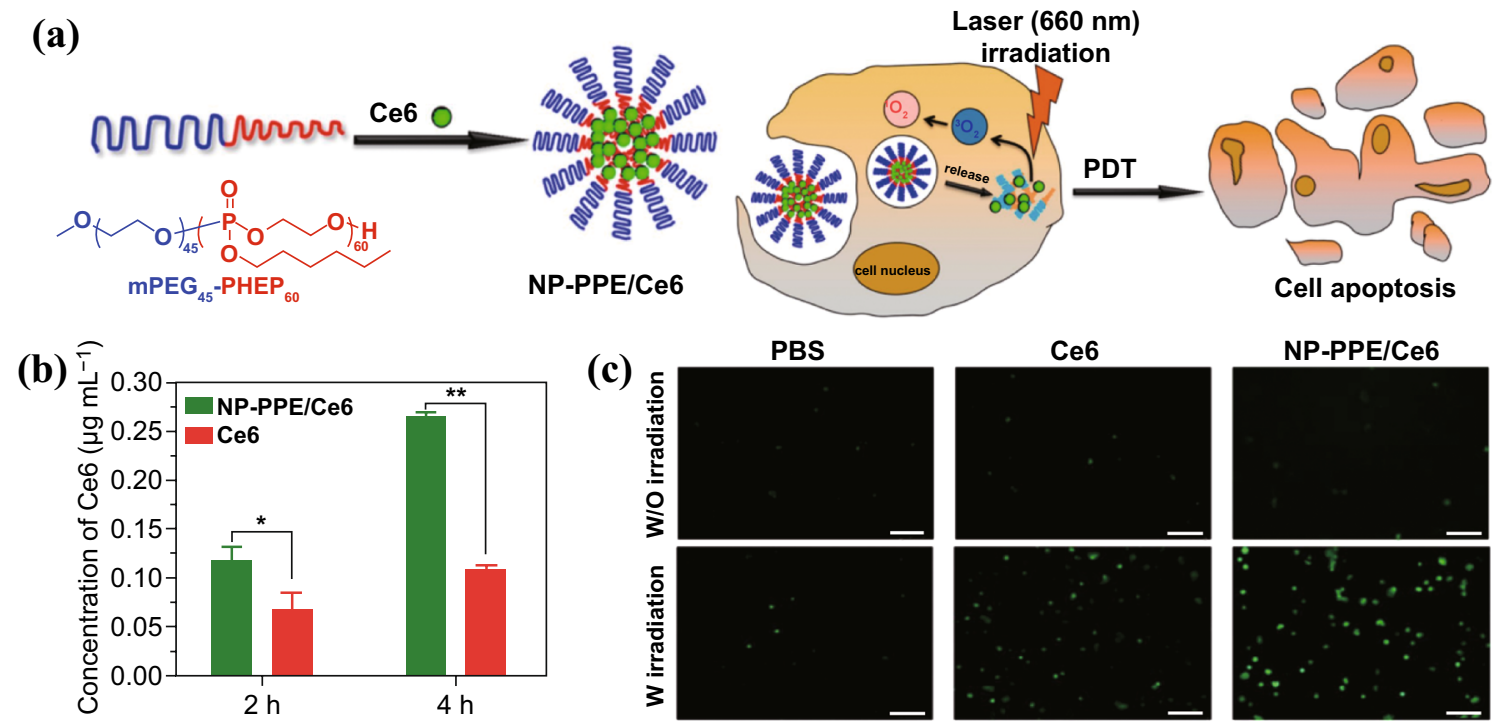

Fig. 2 a Schematic illustration of Ce6 encapsulation by self-assembly of amphiphilic mPEG-b-PHEP and intracellular delivery of Ce6 with NPPPE. b Quantitative Ce6 concentration in the BxPC-3 cancer cells. c CLSM image of cells incubated with DCF and then treated with free Ce6 and NP-PPE/Ce6 with NIR laser irradiation. Adapted with permission from Ref. [36]. Copyright @ 2015, American Chemical Society

were co-loaded within ROS-responsive polymeric vesicles (Fig. 4). Upon $805 \mathrm{~nm}$ laser irradiation, the heat generated by photothermal effect of cypate decomposes $\mathrm{H}_{2} \mathrm{O}_{2}$ into $\mathrm{O}_{2}$, alleviating hypoxic TME in tumor site, as evidenced by weak fluorescence intensity of hypoxia-specific probe pimonidazole and less than $10 \%$ hypoxic areas. Followed by $660 \mathrm{~nm}$ irradiation, Ce6 produced abundant ${ }^{1} \mathrm{O}_{2}$ with the assistance of self-supplied oxygen. Then, the generated ${ }^{1} \mathrm{O}_{2}$ could disrupt the ROS-responsive polymeric vesicles, which subsequent triggered CC-PAMAM diffusing out from vesicular chamber. The released CC-PAMAM was able to penetrate tumors forming a uniform distribution for photodynamic ablation of tumor cells. The involved light treatment procedure comprised five cycles of consecutive irradiations with $805 \mathrm{~nm}$ light for $3 \mathrm{~min}$ and $660 \mathrm{~nm}$ light for $10 \mathrm{~min}$ at $24 \mathrm{~h}$ post-CC-PAMAM/ $/ \mathrm{H}_{2} \mathrm{O}_{2}$ vesicles injection, leading to complete elapse of the BxPC-3 PCa without any regrowth.

The dense collagen network in PCa stroma strongly hinders the intratumoral penetration of oxygen and drugs. Losartan and other angiotensin receptor blockers (ARBs) have been proven to inhibit tumor collagen production via downregulation of TGF- $\beta 1$ [43]. In the AK4.4 pancreatic tumors, losartan treatment was shown to increase drug accumulation by $74 \%$ and oxygen delivery by over onefold. Thus, depletion of collagen with ARBs is a promising strategy to enhance delivery efficiency of PSs and alleviate hypoxia in pancreatic cancer, thereby improving the PDT efficacy. In a study conducted by Li et al. [44], pretreatment with losartan increased the accumulation of PS-loaded nanoparticles by twofold and the tumor growth rates were obviously delayed.

\subsection{Tumor Targeting}

NPs can be targeted to tumor tissues via EPR effect owing to their leaky vasculature and poor lymphatic drainage [45]. In addition, NPs can also be decorated with tumor targeting ligands, such as monoclonal antibodies, antibody fragments, and small molecules, for further enhanced tumor targeting [46-48]. Conjugation to specific ligands or surface receptors in PCa that allows targeted delivery of photosensitizer to PCa cells will remarkably improve the PDT efficacy of PCa treatment. Some potential targets have been suggested for the specific delivery in PCa, such as EGFR, transferrin, epithelial cell adhesion molecule (EpCAM), CD44, CD133, urokinase plasminogen activator receptor (uPAR), ERBB2, and CA125 [49-52]. Er et al. used cetuximab (Cet)modified mesoporous silica nanoparticles (MSNPs) for the tumor targeting delivery of PSs [53]. Cet is a monoclonal antibody of EGFR (epidermal growth factor receptor), which is overexpressed on several PCa types. The Cet decoration facilitates cell recognition and internalization of MSNPs during treatment, which in turn accelerates the release of 


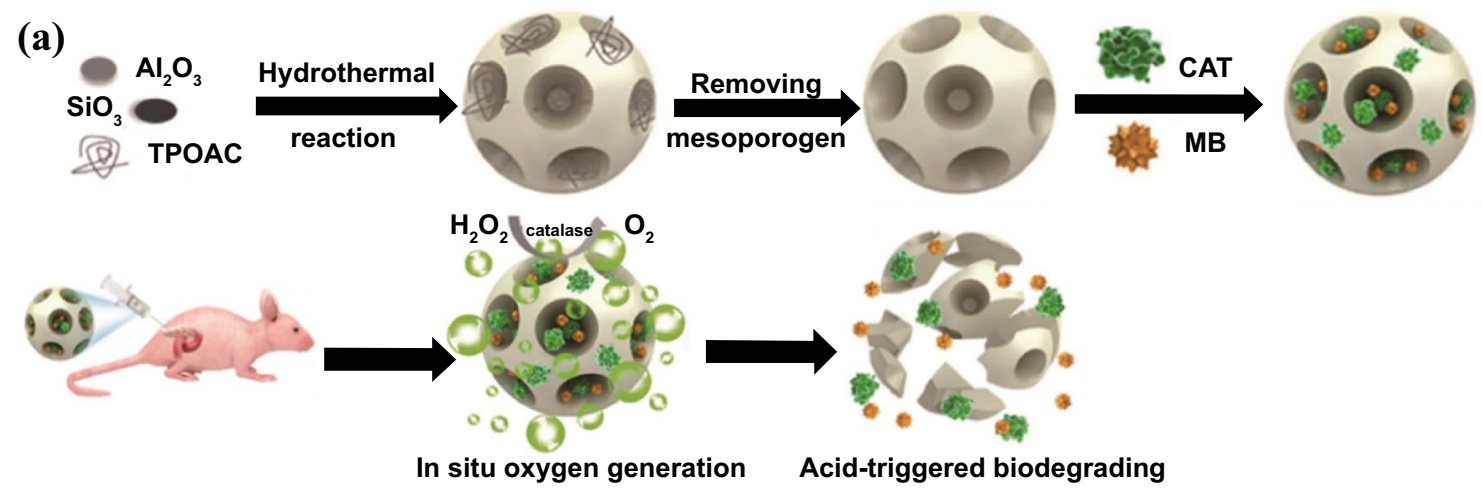

(b)
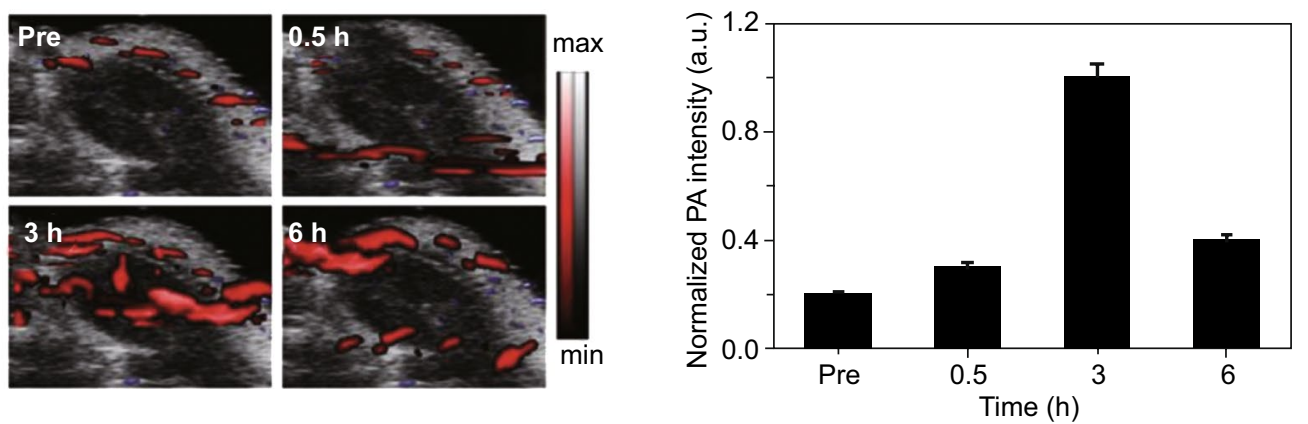

Fig. 3 a Paradigm of the fabrication of mesoporous hierarchical zeolite nanocarriers loading catalase and MB characterized with oxygen generation and acid-triggered degradation. b In vivo 2D photoacoustic images and quantitative results of the blood oxyhemoglobin saturation in the tumor region at different time points after the intratumoral injection of the ZCM nanocapsule. Reproduced from Ref. [40] with permission. Copyright (C) The Royal Society of Chemistry 2018

PSs within cells. A similar Cet-mediated PDT was reported by Obaid et al. [54]. Cet was conjugated on the liposomal formulation of PSs-anchored phospholipid via site-specific Protein $\mathrm{Z}$ tuning approach, and the obtained photoimmunonanoconjugates (PINs) was denoted as Cet-PINs. By optimizing PS lipid anchoring, surface electrostatics, Cet surface orientations, and Cet densities, 16-fold enhancement in binding specificity and targeted photodestruction was achieved. The performance of the Cet-PINs was studied on a stroma-rich heterotypic xenograft model consisting of MIA $\mathrm{PaCa}-2$ cells and pancreatic cancer-associated fibroblasts. Due to the actively homing effect of Cet, the BPD-delivering Cet-PINs achieved rapidly penetration up to $470 \mu \mathrm{m}$ away from vessels within $1 \mathrm{~h}$ in heterotypic PCa organoids. Additionally, Cet-PINs-mediated PDT exhibited excellent tumortargeted photodestruction efficiencies, including a 1.5-fold reduction in tumor collagen density and a statistically significant $\sim 3$-fold increase in fractional necrotic area at 72 -h post-treatment. Remarkably, the used BPD equivalent dose of this Cet-PIN formulation is $\sim 10$-fold lower than that of clinically approved liposomal formulation verteporfin, underscoring the value of molecular targeted PS delivery.

\section{Combining PDT with Other Therapies}

Although PDT is able to kill cancer cells under the optimized condition, tumor relapse is unavoidable in practical scenario with PDT alone. This can be explained by incomplete elimination of cancer cells partly due to the heterogeneous distribution of PSs and light in tumor tissues [42]. In addition, the exacerbated hypoxia induced by PDT also stimulates several signaling pathways leading to cancer cell survival and escape [55]. Therefore, there is a critical need to combine other therapy with PDT for maximized therapeutic efficacy. NPs hold excellent capability to integrate various therapeutic agents together with PSs for PDT-based combinatory treatment. 
(a)
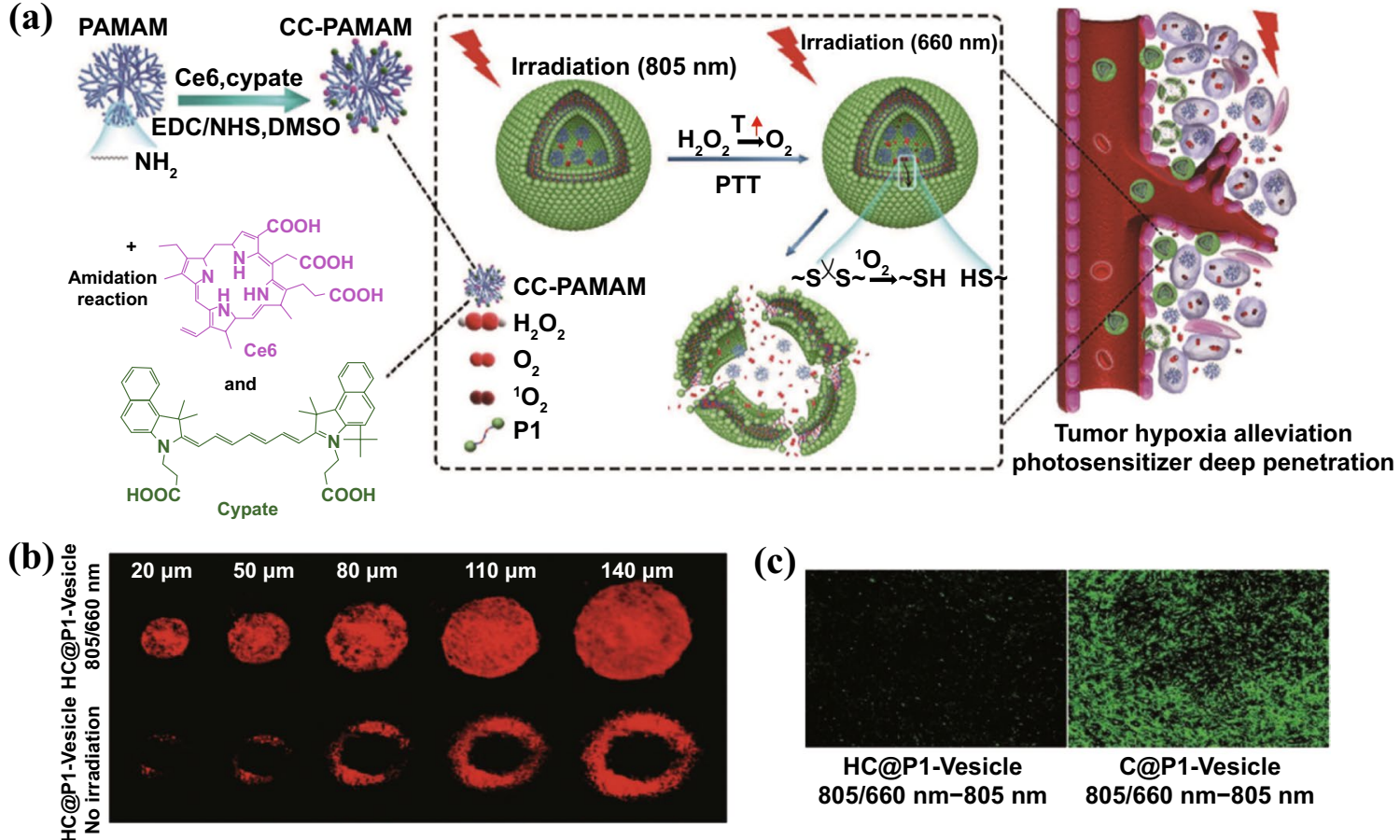

Fig. 4 a Schematic illustration of light-triggered clustered polymeric vesicles with self-supplied oxygen and tissue penetrability for potent PDT against hypoxic PCa tumor. b Penetration profiles of CC-PAMAM after HC@P1-Vesicle treatment with or without 805/660 irradiation in multicellular tumor spheroids. The imaging was conducted at 2-h postirradiation using CLSM. c Hypoxia of BxPC-3 tumor treated with C@P1-Vesicle or HC@P1-Vesicle using a hypoxia-specific probe pimonidazole (hypoxyprobe-1 plus kit). HC@P1-Vesicle: $\mathrm{H}_{2} \mathrm{O}_{2}$ and CC-PAMAM-loaded ROS-responsive P1 polymeric vesicles. C@P1-Vesicle: CC-PAMAM-loaded P1 polymeric vesicles without $\mathrm{H}_{2} \mathrm{O}_{2}$. Reproduced from Ref. [42] with permission. Copyright @ 2017 WILEY-VCH Verlag GmbH \& Co. KGaA, Weinheim

\subsection{Combining PDT with Photothermal Therapy}

Photothermal therapy (PTT) makes use of photothermal agents that can convert the absorbed light energy into heat to increase the temperature of surrounding environment and trigger the death of cancer cells [56-58]. The combined therapy with PDT and PTT is attractive in treating cancer, because the cytotoxic ${ }^{1} \mathrm{O}_{2}$ and hyperthermia are produced by PDT/PTT sensitizers in similar light triggering conditions $[59,60]$. Besides, many light-responsive nanomaterials can absorb photons from NIR laser irradiation and then generate both heat and ${ }^{1} \mathrm{O}_{2}$ relying on different non-fluorescent excited state relaxation pathways that can function in parallel [61, 62]. Examples of such dual functional materials include inorganic species (e.g., AuNPs, black phosphorus, graphene oxide, and $\mathrm{Ti}_{3} \mathrm{C}_{2}$ nanosheets) and organic species (e.g., diketopyrrolopyrroles, heptamethine dyes (indocyanine green, IR780, IR825, IR808, IR2), and metallonaphthalocyanines) [59, 61, 63, 64]. Moreover, the photothermal conversion is oxygen-independent, which makes PTT an excellent compensate for oxygen-dependent PDT, especially in hypoxic tumors [65]. However, PTT alone is unlikely to eliminate all tumor cells because the resulting heat can be rapidly dissipated by circulating blood $[66,67]$. Therefore, combined PTT/PDT treatment can enhance the outcome of cancer therapy by compensating for the drawbacks of monotherapies [68].

Yin and coworkers developed the biodegradable PEGylated oxygen-deficient molybdenum oxide nanoparticles (PEG-MoOx NPs), which could effectively convert light into heat and generate ${ }^{1} \mathrm{O}_{2}$ simultaneously with a single 1064 nm NIR irradiation [69]. The PEG-MoOx NPs were synthesized through a one-pot hydrothermal process using PEG-4000 and $\left(\mathrm{NH}_{4}\right)_{6} \mathrm{Mo}_{7} \mathrm{O}_{24} \cdot 4 \mathrm{H}_{2} \mathrm{O}$ hydrolyzed in water-ethanol solution $(\mathrm{pH}=1.2)$. The mechanism of ${ }^{1} \mathrm{O}_{2}$ production was speculated to be increased thermionic electron emission of PEG-MoOx NPs caused by extreme heat development upon $1064 \mathrm{~nm}$ NIR activation. The synergistic 
PDT/PTT effect of PEG-MoOx NPs with 1064 nm irradiation caused a far better tumor therapy effect in PANC-1 bearing mice at a lower light dose $\left(0.6 \mathrm{~W} \mathrm{~cm}^{-2}\right)$, compared with sole PTT effect of PEG-MoOx NPs induced by $808 \mathrm{~nm}$ laser $\left(0.75 \mathrm{~W} \mathrm{~cm}^{-2}\right)$.

Li et al. applied a distinct strategy for combinatorial PDT/ PTT treatment against PCa, wherein gold nanoparticles (AuNPs) were employed to not only deliver PSs but also act as photothermal agents [70]. The photosensitizer prodrug 5-aminolevulinic acid (5-ALA) was linked with peptide CRQAGFSL, which can be cleaved by tumor-specific intracellular CathepsinE (CTSE), to realize targeting release of PSs within tumor. The CRQAGFSL-5-ALA conjugated AuNPs were cross-linked with 1,9-nonanedithiol forming spherical gold nanoclusters. In addition, an active targeting U11 peptide was also conjugated on the surface of nanocluster. This multifunctional nanocluster-based platform represents a promising PDT/PTT agent for highly synergistic therapeutic effect against $\mathrm{PCa}$ with reduced side effects in normal pancreas tissues.

\subsection{Combining PDT with Other ROS-Generating Therapy}

For ROS-dependent anticancer treatment, a relatively high level of ROS is required to induce irreversible oxidative damage, because cancer cells can protect themselves from oxidative stress through the overexpressed intracellular reducing thiol species [71]. However, the ROS generation efficiency of PDT is largely limited to the low tissue oxygen in pancreatic cancers. In addition, PSs widely used in clinic generally absorb light in the visible red range [72]. Even though NIR light can penetrate deeper than visible light, it can only reach around $3 \mathrm{~cm}$ deep in tissue [59]. This limits clinical applications of PDT, especially in large tumors. The combination of PDT with other ROS-generating therapy is a promising approach for enhanced ROS-mediated therapeutic outcome.

Recently, the combination of PDT and chemodynamic therapy (CDT) has been continuously explored to amplify the tumor oxidative stress [73, 74]. The therapeutic mechanism of CDT lies in the catalysis of metal ions-based nanoparticles to convert endogenous $\mathrm{H}_{2} \mathrm{O}_{2}$ into cytotoxic hydroxyl radicals $(\mathrm{OH})$ via Fenton-like reaction [39]. Although CDT is an unremitting chemical process overcoming the limitations of light attenuation and hypoxic TME [75], the efficacy of CDT is always restricted by the unsatisfactory catalytic efficiency of currently developed metal-based nanocatalysts [39]. The extent of acidity in TME also restricts the activity of these metallic catalysts, which obviously decreases CDT performance [76]. Additionally, the concentration of $\mathrm{H}_{2} \mathrm{O}_{2}$ in tumor tissue $(<100 \mu \mathrm{M})$ is still insufficient to generate enough $\mathrm{OH}$ for effective CDT [77]. For all of these, CDT alone still cannot achieve an envisioned therapeutic outcome in intractable PCa. However, the encouraging results have been obtained with dual-modal PDT/CDT with better therapeutic effect than CDT or PDT alone [78]. A recent study by Li et al. [44] is a good example of such combined PDT/CDT in treating $\mathrm{PCa}$, where they employed hollow mesoporous organosilica nanoparticle (HMON) to co-deliver PDT/CDT agents so as to produce ${ }^{1} \mathrm{O}_{2}$ and $\mathrm{OH}$ in response to laser irradiation and tumor $\mathrm{H}_{2} \mathrm{O}_{2}$ for enhanced antitumor effects. The photosensitizer HPPH was incorporated in the framework of HMON through cohydrolysis of HPPH-silane and silane precursors, and the hollow cavity of HMON was exploited as a nanoreactor for in situ polymerization to immobilize ultrasmall Au NPs $(<3.6 \mathrm{~nm})$ via the chelation effect (Fig. 5). These ultrasmall Au NPs behaving like glucose oxidase could catalyze glucose into $\mathrm{H}_{2} \mathrm{O}_{2}$ to provide self-supplied $\mathrm{H}_{2} \mathrm{O}_{2}$ for CDT. CDT agents $\mathrm{Cu}^{2+}$-tannic acid (Cu-TA) complexes were deposited on the surface of HMON-Au, which can catalyze the self-supplied $\mathrm{H}_{2} \mathrm{O}_{2}$ into $\mathrm{OH}$ in acidic TME. Collagenase (Col) was further loaded into the HMONs$\mathrm{Au} @ \mathrm{Cu}-\mathrm{TA}$ to degrade the dense stroma within PCa thereby enhancing the deep penetration of HMONs and promoting $\mathrm{O}_{2}$ infiltration to alleviate hypoxia. The combined PDT/CDT produced large amount of intracellular ROS in BxPC-3 cells and eliminated the tumors on BxPC- 3 bearing mice, superior to either monotherapy.

Sonodynamic therapy (SDT) is another ROS-dependent treatment, referring to the use of low intensity ultrasound to activate sonosensitizers, which converts tissue oxygen to cytotoxic ${ }^{1} \mathrm{O}_{2}$ [71, 79-81]. To date, almost all the sensitizers used in SDT based studies were originally used as photosensitizers [82]. Due to the higher tissues penetration of ultrasound, combining SDT with traditional PDT can make up the tissue penetration restriction of light. Besides, sonophotodynamic therapy (SPDT) can decrease the necessary dosage of sensitizer and light energy of PDT, which in turn further reduces its off-target photocytotoxicity [83]. Indeed, 
SPDT treatment using sono/photosensitizers which can be activated by light plus US irradiation has been tested with enhanced ROS generation and better therapeutic outcomes than SDT or PDT alone in various tumor types [84-87]. Wang et al. [86] investigated Chlorin e6 (Ce6)-mediated SPDT (Ce6-SPDT) on 4T1 cells and animal models. Much more ${ }^{1} \mathrm{O}_{2}$ generation was observed in cells treated by SPDT compared with PDT or SDT alone (MFI = 156 in SPDT vs $\mathrm{MFI}=51$ in PDT/MFI $=10$ in SDT, mean fluorescence intensity). In addition, Ce6-SPDT markedly inhibited tumor growth (volume and weight) and lung metastasis in 4T1 tumor-bearing mice. The synergistic effects of PDT/ SDT observed in other types of cancer should be applicable to pancreatic cancer. A recent study by Chen et al. showed a rational design of HMONs-based oxygen-loaded nanoplatform for SDT treatment on PCa models [88]. The mesoporous structure of HMONs was served as IR780 carrier. Meanwhile, HMONs were chelated with fluorocarbon (FC)-chains, which served as oxygen binding sites for exogenous oxygen delivery. IR780 has been reported for producing ${ }^{1} \mathrm{O}_{2}$ under irradiation with $808 \mathrm{~nm}$ laser [89]. Therefore, theoretically, this IR780@ $\mathrm{O}_{2}$-FHMON is promising for exerting IR780-mediated PDT/SDT synergistic effect, which can be further enhanced by self-supplying oxygen.

\subsection{Multimodal Synergistic Therapy Based on PDT}

Given the aggressive nature from an early stage and the multiple escape mechanisms of pancreas cancer, any monotherapy is unlikely to cure cancer completely [90]. Thus, tumor relapse and metastasis occurred in several cases after sole PDT treatment $[12,15]$. Thus, the appropriately conceived combination strategies based on PDT are essential for this extremely difficult-to-treat cancer, such that therapeutics targeting distinct tumor compartments or signaling pathways could induce synergistic effects to overcome stubborn $\mathrm{PCa}$ resistance.

The combination of chemotherapy and PDT has shown promising synergistic effect in various cancer types including $\mathrm{PCa}$ both in the preclinical and clinical studies [91-93]. Zhang et al. conjugated $\mathrm{Fe}^{3+}$ with photosensitizer 5,10,15,20-tetra(p-benzoato)porphyrin (TBP) to create a

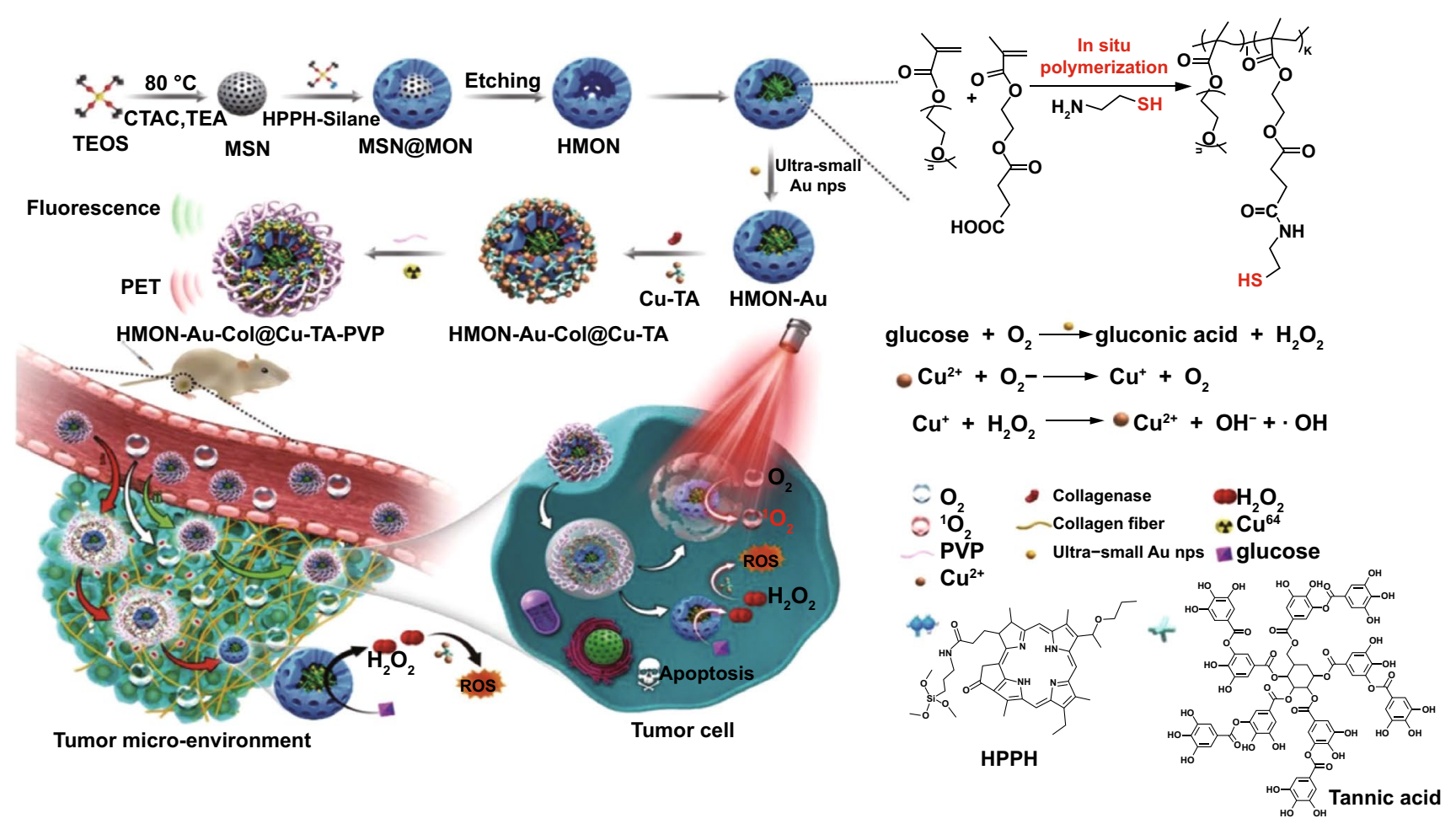

Fig. 5 Schematic showing the fabrication process of HMONs-Au@Cu-TA and its application for synergistic PDT/CDT therapy. Reprinted from Ref. [44] with permission. Copyright @ 2019 WILEY-VCH Verlag GmbH \& Co. KGaA, Weinheim 
porous coordination network (PCN) [94]. This Fe(III)-TBP PCN was able to load paclitaxel (PTX) and release it in response to both laser irradiation and $\mathrm{pH}$ changes within tumors. Besides, catalase-like $\mathrm{Fe}^{3+}$ could catalyze endogenous $\mathrm{H}_{2} \mathrm{O}_{2}$ decomposed into $\mathrm{O}_{2}$ to support PDT activity. Thus, a single nanoplatform-mediated synergistic effect of chemo/PDT was achieved in PANC-1 tumors, where phaseblocked cells were accelerated in transforming into apoptotic state upon PDT introduction.

In addition to directly killing tumor cells, PDT can also induce microvascular shutdown, which contribute to exacerbated hypoxia in TME and nutrients deprivation. Although the vascular damage can induce tumor death, the increased tumor hypoxia also stimulates several signaling pathways leading to angiogenesis and tumor metastasis [5, 95]. To solve this issue, Spring et al. introduced a photoactivatable multi-inhibitor nanoliposome (PMIL) for combining PDT with cabozantinib (XL184), a multikinase inhibitor, which targets vascular endothelial growth factor (VEGF) signaling and MET - the receptor tyrosine kinase for hepatocyte growth factor-signaling (Fig. 6) [55]. VEGF signaling promotes tumor angiogenesis and vascular regrowth, while MET signaling supports cancer cell survival and promotes cancer cell metastatic escape from hypoxic tumor induced by cytotoxic and vascular damage therapy [96]. PMIL is fabricated by encapsulating XL184-loaded poly(lactic acidco-glycolic) acid (PLGA) NPs in BPD-loaded cationic liposome. Upon irradiation with $690 \mathrm{~nm}$ laser, the light-activated ROS generation by BPD induced not only tumor cell death and microvessel damage, but also liposomal disruption to release XL184-loaded PLGA NPs. The PLGA NPs were designed for sustained release of XL184 over a period of several days. XL184 inhibits both the VEGF and MET signaling pathways to suppress tumor escape. The prominent therapeutic efficacy of PMIL was validated on two PCa xenograft model with significantly enhanced inhibition in both primary and metastatic tumor.

\section{Conclusion and Outlook}

The efficacy of PDT on alleviating tumor burden in unresectable localized PCa patients have been validated in several clinical trials, with relatively lower complications [12, 15-17]. Under the guidance of ultrasound imaging and/or $\mathrm{CT}$, optical fibers can be inserted into the pancreas tumors in a percutaneous or transduodenal approach to realize minimal-invasive photodynamic tumor ablation. Despite the promising results obtained in the preliminary clinical trials, the efficacy of PDT is largely compromised by lack of suitable PSs in terms of tumor targeting, ${ }^{1} \mathrm{O}_{2}$ yield, and adaptability to hypoxic TME. In this regard, NPs-based PSs have shown the promising results to solve these issues in various preclinical researches. Rationally designed nanocarriers can load PSs with very high loading content while avoiding potential aggregation and improving ${ }^{1} \mathrm{O}_{2}$ generation [31-33]. To further improve the ${ }^{1} \mathrm{O}_{2}$ generation in hypoxic tumor, PSs-loaded NPs can also be designed for oxygen delivery or in situ oxygen generation [38]. In addition, the NPs inherently can be targeted to tumors via EPR effect or modified with specific ligands to achieve active tumor targeting [41]. Moreover, since PDT alone is not enough to cure PCa completely, various therapeutic agents can also be co-loaded with PSs in NPs to synchronize with PDT for maximized therapeutic efficacy $[5,55,95]$.

Although NPs-based PDT have achieved far better therapeutic efficacy than conventional PSs for PCa therapy in the preclinical studies, the translation of NPs-based PDT from bench to bedside is not straightforward. There are still several issues to address for the clinical translation of NPs-based PDT. On the one hand, various aspects in the NPs design need to be optimized in respect to PSs loading, surface charge, size, targeting modification, etc. [54], as these factors determine the extent of interactions between NPs and biomolecules, and are crucial in pharmacology and clearance rate of NPs. On the other hand, although integrating various therapeutic components in one NP benefits cancer therapy in preclinical studies, their usually sophisticated manufacturing process may complicate the potential pharmaceutical development in terms of quality control and reproducibility. In addition, most of the preclinical studies are conducted in tumor xenograft model of mice and there is still a lack of researches on large animals or other clinicalrelevant models. The potential safety issues and consistency of therapeutic efficacy are the major concerns. For the purpose of successful clinical translation of synthesized NPs, the detailed biodistribution investigation and excretion assay are highly desired to evidence their good biocompatibility and biodegradability. Repeated permutation experiments for certain NPs should be carried out to establish optimum treatment parameters such as light dose, interval time between PS administration and irradiation, PS dose and administration 
(a)

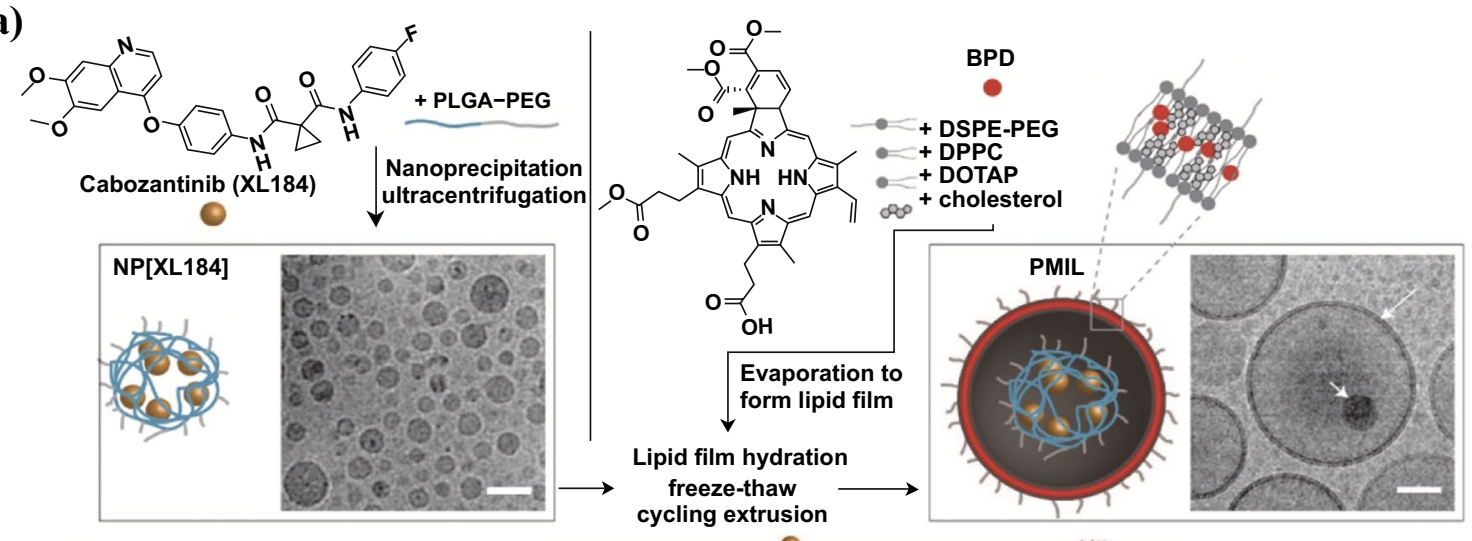

(b)

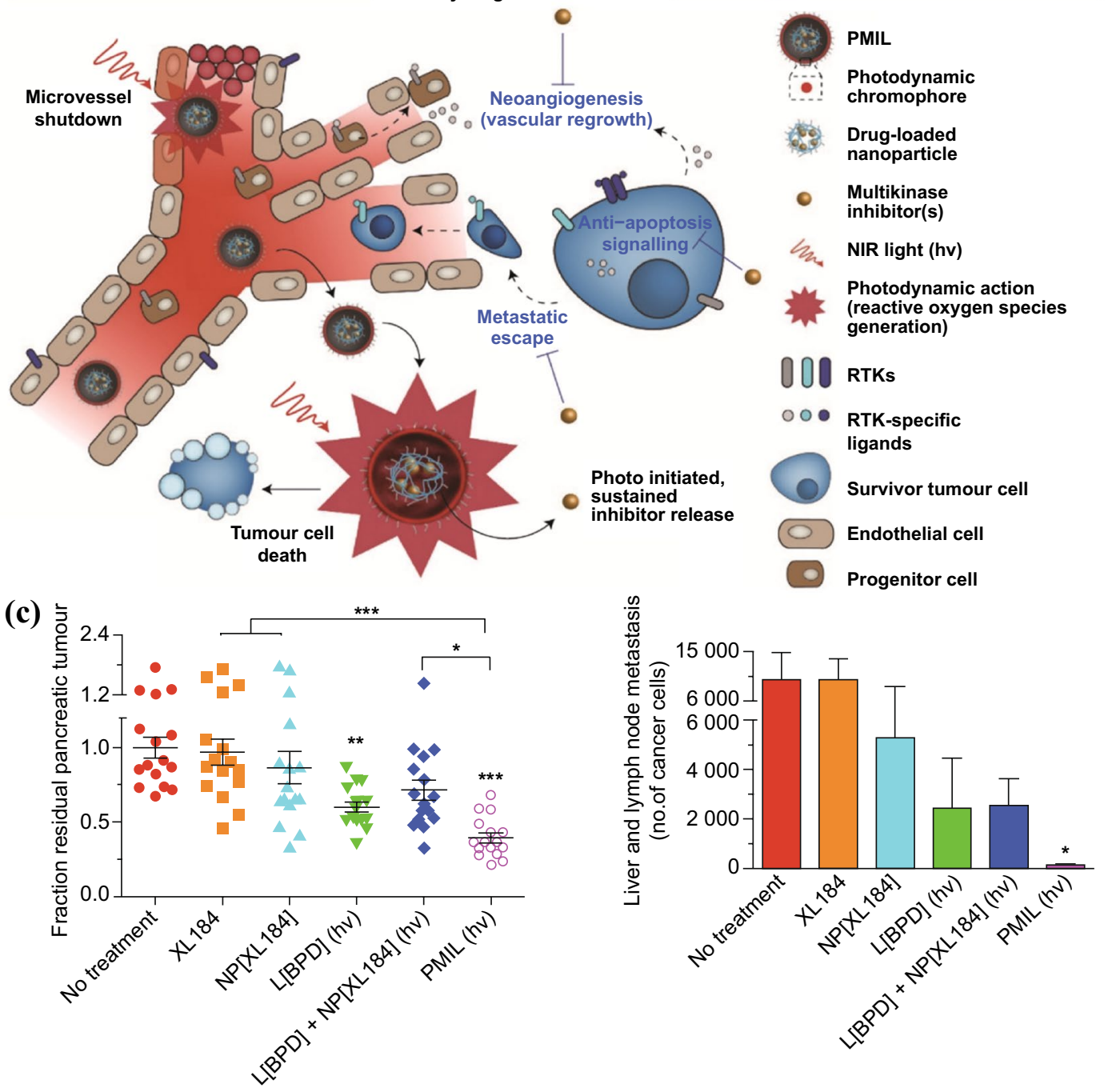

Fig. 6 Schematic illustration of a fabrication of a photoactivatable multi-inhibitor nanoliposome (PMIL) and $\mathbf{b}$ its applications for combination therapy with PDT-induced tumor cell death and microvessel damage and inhibition of treatment escape pathways. c A single PMIL treatment in orthotopic PDAC mouse achieves enhanced reductions in primary (Left) and metastatic tumors (Right). Reproduced from Ref. [55] with permission. Copyright (C) 2016 Macmillan Publishers Limited 
method, so as to guide future clinical translation. Collectively, the present studies have demonstrated the potential of NPs-based PDT for PCa therapy and further studies are warranted to optimize NPs design and investigate the longterm safety and efficacy. The existing clinical studies and continuing phase II/III studies of PDT in PCa form a good basis for developing NPs-based PDT against PCa.

Acknowledgements This contribution was financially supported by Beijing Natural Science Foundation, Haidian, original innovation joint fund (No. 17L20170), National Key Research and Development Program of China (No. 2016YFA0201400), State Key Program of National Natural Science of China (No. 81930047), Projects of International Cooperation and Exchanges NSFC-PSF (No. 31961143003), National Project for Research and Development of Major Scientific Instruments (No. 81727803), and the Foundation for Innovative Research Groups of the National Natural Science Foundation of China (No. 81421004).

Open Access This article is licensed under a Creative Commons Attribution 4.0 International License, which permits use, sharing, adaptation, distribution and reproduction in any medium or format, as long as you give appropriate credit to the original author(s) and the source, provide a link to the Creative Commons licence, and indicate if changes were made. The images or other third party material in this article are included in the article's Creative Commons licence, unless indicated otherwise in a credit line to the material. If material is not included in the article's Creative Commons licence and your intended use is not permitted by statutory regulation or exceeds the permitted use, you will need to obtain permission directly from the copyright holder. To view a copy of this licence, visit http://creativecommons.org/licenses/by/4.0/.

\section{References}

1. R.L. Siegel, K.D. Miller, A. Jemal, Cancer statistics, 2019. CA Cancer J. Clin. 69, 7-34 (2019). https://doi.org/10.3322/ caac. 21551

2. J.P. Neoptolemos, J. Kleeff, P. Michl, E. Costello, W. Greenhalf et al., Therapeutic developments in pancreatic cancer: current and future perspectives. Nat. Rev. Gastroenterol. Hepatol. 15, 333-348 (2018). https://doi.org/10.1038/s4157 5-018-0005-x

3. M.G. Keane, K. Bramis, S.P. Pereira, G.K. Fusai, Systematic review of novel ablative methods in locally advanced pancreatic cancer. World J. Gastroenterol. 20, 2267-2278 (2014). https://doi.org/10.3748/wjg.v20.i9.2267

4. T. Conroy, F. Desseigne, M. Ychou, O. Bouche, R. Guimbaud et al., FOLFIRINOX versus gemcitabine for metastatic pancreatic cancer. New Engl. J. Med. 364, 1817-1825 (2011). https ://doi.org/10.1056/NEJMoa1011923

5. S. Tangutoori, B.Q. Spring, Z. Mai, A. Palanisami, L.B. Mensah et al., Simultaneous delivery of cytotoxic and biologic therapeutics using nanophotoactivatable liposomes enhances treatment efficacy in a mouse model of pancreatic cancer. Nanomedicine 12, 223-234 (2016). https://doi.org/10.1016/j. nano.2015.08.007

6. H.C. Huang, S. Mallidi, J. Liu, C.T. Chiang, Z. Mai et al., Photodynamic therapy synergizes with irinotecan to overcome compensatory mechanisms and improve treatment outcomes in pancreatic cancer. Cancer Res. 76, 1066-1077 (2016). https ://doi.org/10.1158/0008-5472.CAN-15-0391

7. M. Sivasubramanian, Y.C. Chuang, L.W. Lo, Evolution of nanoparticle-mediated photodynamic therapy: from superficial to deep-seated cancers. Molecules 24, 520 (2019). https://doi. org/10.3390/molecules24030520

8. P.N. Manghnani, W. Wu, S. Xu, F. Hu, C. The et al., Visualizing photodynamic therapy in transgenic zebrafish using organic nanoparticles with aggregation-induced emission. Nano-Micro Lett. 10, 61 (2018). https://doi.org/10.1007/s4082 0-018-0214-4

9. S.S. Jalde, A.K. Chauhan, J.H. Lee, P.K. Chaturvedi, J.S. Park et al., Synthesis of novel Chlorin e6-curcumin conjugates as photosensitizers for photodynamic therapy against pancreatic carcinoma. Eur. J. Med. Chem. 147, 66-76 (2018). https://doi. org/10.1016/j.ejmech.2018.01.099

10. S.W. Tait, D.R. Green, Mitochondria and cell death: outer membrane permeabilization and beyond. Nat. Rev. Mol. Cell Biol. 11, 621-632 (2010). https://doi.org/10.1038/nrm2952

11. H. Su, Z. Li, L. Lazar, Y. Alhamoud, X. Song et al., In vitro evaluation of the toxicity and underlying molecular mechanisms of Janus $\mathrm{Fe}_{3} \mathrm{O}_{4}-\mathrm{TiO}_{2}$ nanoparticles in human liver cells. Environ. Toxicol. 33, 1078-1088 (2018). https://doi. org/10.1002/tox.22631

12. S.G. Bown, A.Z. Rogowska, D.E. Whitelaw, W.R. Lees, L.B. Lovat et al., Photodynamic therapy for cancer of the pancreas. Gut 50, 549-557 (2002). https://doi.org/10.1136/gut.50.4.549

13. S.K. Rajendrakumar, S. Uthaman, C.S. Cho, I.K. Park, Nanoparticle-based phototriggered cancer immunotherapy and its domino effect in the tumor microenvironment. Biomacromolecules 19, 1869-1887 (2018). https://doi.org/10.1021/acs. biomac. 8 b00460

14. M. Saeed, W. Ren, A. Wu, Therapeutic applications of iron oxide based nanoparticles in cancer: basic concepts and recent advances. Biomater. Sci. 6, 708-725 (2018). https:// doi.org/10.1039/c7bm00999b

15. M.T. Huggett, M. Jermyn, A. Gillams, R. Illing, S. Mosse et al., Phase I/II study of verteporfin photodynamic therapy in locally advanced pancreatic cancer. Br. J. Cancer 110, 16981704 (2014). https://doi.org/10.1038/bjc.2014.95

16. J.-H. Choi, D. Oh, J. Lee, J.-H. Park, K.-P. Kim et al., Initial human experience of endoscopic ultrasound-guided photodynamic therapy with a novel photosensitizer and a flexible laser-light catheter. Endoscopy 47, 1035-1038 (2015). https ://doi.org/10.1055/s-0034-1392150

17. J.M. DeWitt, K. Sandrasegaran, B. O’Neil, M.G. House, N.J. Zyromski et al., Phase 1 study of EUS-guided photodynamic therapy for locally advanced pancreatic cancer. Gastrointest. Endosc. 89, 390-398 (2019). https://doi.org/10.1016/j. gie.2018.09.007 
18. L. Chambre, W.S. Saw, G. Ekineker, L.V. Kiew, W.Y. Chong et al., Surfactant-free direct access to porphyrin-cross-linked nanogels for photodynamic and photothermal therapy. Bioconjug. Chem. 29, 4149-4159 (2018). https://doi.org/10.1021/acs. bioconjchem. $8 \mathrm{~b} 00787$

19. F. Hu, S. Xu, B. Liu, Photosensitizers with aggregationinduced emission: materials and biomedical applications. Adv. Mater. 30, e1801350 (2018). https://doi.org/10.1002/ adma.201801350

20. Y. Wang, G. Wei, X. Zhang, F. Xu, X. Xiong et al., A stepby-step multiple stimuli-responsive nanoplatform for enhancing combined chemo-photodynamic therapy. Adv. Mater. 29, 1605357 (2017). https://doi.org/10.1002/adma.201605357

21. K.A. Carter, D. Luo, A. Razi, J. Geng, S. Shao et al., Sphingomyelin liposomes containing porphyrin-phospholipid for irinotecan chemophototherapy. Theranostics 6, 2329-2336 (2016). https://doi.org/10.7150/thno.15701

22. D. Luo, K.A. Carter, A. Razi, J. Geng, S. Shao et al., Doxorubicin encapsulated in stealth liposomes conferred with lighttriggered drug release. Biomaterials 75, 193-202 (2016). https ://doi.org/10.1016/j.biomaterials.2015.10.027

23. R. Liu, J. Tang, Y. Xu, Z. Dai, Bioluminescence imaging of inflammation in vivo based on bioluminescence and fluorescence resonance energy transfer using nanobubble ultrasound contrast agent. ACS Nano 13, 5124-5132 (2019). https://doi. org/10.1021/acsnano.8b08359

24. C. Gao, P. Bhattarai, M. Chen, N. Zhang, S. Hameed et al., Amphiphilic drug conjugates as nanomedicines for combined cancer therapy. Bioconjug. Chem. 29, 3967-3981 (2018). https://doi.org/10.1021/acs.bioconjchem.8b00692

25. C. Gao, X. Liang, S. Mo, N. Zhang, D. Sun et al., Near-infrared cyanine-loaded liposome-like nanocapsules of camptothecin-floxuridine conjugate for enhanced chemophotothermal combination cancer therapy. ACS Appl. Mater. Interfaces 10, 3219-3228 (2018). https://doi.org/10.1021/acsami.7b14125

26. R.A. Day, D.A. Estabrook, J.K. Logan, E.M. Sletten, Fluorous photosensitizers enhance photodynamic therapy with perfluorocarbon nanoemulsions. Chem. Commun. 53, 1304313046 (2017). https://doi.org/10.1039/c7cc07038a

27. H. Hu, X. Yan, H. Wang, J. Tanaka, M. Wang et al., Perfluorocarbon-based $\mathrm{O}_{2}$ nanocarrier for efficient photodynamic therapy. J. Mater. Chem. B 7, 1116-1123 (2019). https://doi. org/10.1039/c8tb01844h

28. N. Yang, W. Xiao, X. Song, W. Wang, X. Dong, Recent advances in tumor microenvironment hydrogen peroxideresponsive materials for cancer photodynamic therapy. NanoMicro Lett. 12, 15 (2020). https://doi.org/10.1007/s4082 0-019-0347-0

29. S.K. Golombek, J.N. May, B. Theek, L. Appold, N. Drude et al., Tumor targeting via EPR: strategies to enhance patient responses. Adv. Drug Deliv. Rev. 130, 17-38 (2018). https:// doi.org/10.1016/j.addr.2018.07.007

30. J. Yoo, C. Park, G. Yi, D. Lee, H. Koo, Active targeting strategies using biological ligands for nanoparticle drug delivery systems. Cancers 11, 640 (2019). https://doi.org/10.3390/ cancers 11050640
31. S. Hameed, P. Bhattarai, X. Liang, N. Zhang, Y. Xu et al., Selfassembly of porphyrin-grafted lipid into nanoparticles encapsulating doxorubicin for synergistic chemo-photodynamic therapy and fluorescence imaging. Theranostics 8, 5501-5518 (2018). https://doi.org/10.7150/thno.27721

32. X. Liang, X. Li, L. Jing, X. Yue, Z. Dai, Theranostic porphyrin dyad nanoparticles for magnetic resonance imaging guided photodynamic therapy. Biomaterials 35, 6379-6388 (2014). https://doi.org/10.1016/j.biomaterials.2014.04.094

33. Y. You, X. Liang, T. Yin, M. Chen, C. Qiu et al., Porphyrin-grafted lipid microbubbles for the enhanced efficacy of photodynamic therapy in prostate cancer through ultrasoundcontrolled in situ accumulation. Theranostics 8, 1665-1677 (2018). https://doi.org/10.7150/thno.22469

34. X. Liang, X. Li, X. Yue, Z. Dai, Conjugation of porphyrin to nanohybrid cerasomes for photodynamic diagnosis and therapy of cancer. Angew. Chem. Int. Ed. 50, 11622-11627 (2011). https://doi.org/10.1002/anie.201103557

35. X. Liang, M. Chen, P. Bhattarai, S. Hameed, Z. Dai, Perfluorocarbon@porphyrin nanoparticles for tumor hypoxia relief to enhance photodynamic therapy against liver metastasis of colon cancer. ACS Nano (2020). https://doi.org/10.1021/acsna no.0c05617

36. F. Ding, H.J. Li, J.X. Wang, W. Tao, Y.H. Zhu et al., Chlorin e6-encapsulated polyphosphoester based nanocarriers with viscous flow core for effective treatment of pancreatic cancer. ACS Appl. Mater. Interfaces 7, 18856-18865 (2015). https:// doi.org/10.1021/acsami.5b05724

37. D.P. Ryan, T.S. Hong, N. Bardeesy, Pancreatic adenocarcinoma. N. Engl. J. Med. 371, 1039-1049 (2014). https://doi. org/10.1056/NEJMra1404198

38. Y. Cheng, H. Cheng, C. Jiang, X. Qiu, K. Wang et al., Perfluorocarbon nanoparticles enhance reactive oxygen levels and tumour growth inhibition in photodynamic therapy. Nat. Commun. 6, 8785 (2015). https://doi.org/10.1038/ncomms9785

39. Y. Shi, J. Zhang, H. Huang, C. Cao, J. Yin et al., Fe-doped polyoxometalate as acid-aggregated nanoplatform for NIRII photothermal-enhanced chemodynamic therapy. Adv. Healthc. Mater. 9, e2000005 (2020). https://doi.org/10.1002/ adhm. 202000005

40. D. Hu, Z. Chen, Z. Sheng, D. Gao, F. Yan et al., A catalaseloaded hierarchical zeolite as an implantable nanocapsule for ultrasound-guided oxygen self-sufficient photodynamic therapy against pancreatic cancer. Nanoscale 10, 17283-17292 (2018). https://doi.org/10.1039/c8nr05548c

41. S. Kang, Y.G. Gil, D.H. Min, H. Jang, Nonrecurring circuit nanozymatic enhancement of hypoxic pancreatic cancer phototherapy using speckled Ru-Te hollow nanorods. ACS Nano 14, 4383-4394 (2020). https://doi.org/10.1021/acsnano.9b099 74

42. J. Li, K. Wei, S. Zuo, Y. Xu, Z. Zha et al., Light-triggered clustered vesicles with self-supplied oxygen and tissue penetrability for photodynamic therapy against hypoxic tumor. Adv. Funct. Mater. 27, 1702108 (2017). https://doi.org/10.1002/ adfm.201702108 
43. V.P. Chauhan, J.D. Martin, H. Liu, D.A. Lacorre, S.R. Jain et al., Angiotensin inhibition enhances drug delivery and potentiates chemotherapy by decompressing tumour blood vessels. Nat. Commun. 4, 2516 (2013). https://doi. org/10.1038/ncomms3516

44. L. Li, Z. Yang, W. Fan, L. He, C. Cui et al., In situ polymerized hollow mesoporous organosilica biocatalysis nanoreactor for enhancing ROS-mediated anticancer therapy. Adv. Funct. Mater. 30, 1907716 (2019). https://doi.org/10.1002/ adfm.201907716

45. Y. Liu, H. Miyoshi, M. Nakamura, Nanomedicine for drug delivery and imaging: a promising avenue for cancer therapy and diagnosis using targeted functional nanoparticles. Int. J. Cancer 120, 2527-2537 (2007). https://doi.org/10.1002/ ijc. 22709

46. P.P. Adiseshaiah, R.M. Crist, S.S. Hook, S.E. McNeil, Nanomedicine strategies to overcome the pathophysiological barriers of pancreatic cancer. Nat. Rev. Clin. Oncol. 13, 750-765 (2016). https://doi.org/10.1038/nrclinonc.2016.119

47. Z. Gong, M. Chen, Q. Ren, X. Yue, Z. Dai, Fibronectintargeted dual-acting micelles for combination therapy of metastatic breast cancer. Signal Transduct. Target Ther. 5, 12 (2020). https://doi.org/10.1038/s41392-019-0104-3

48. R.K. Jain, T. Stylianopoulos, Delivering nanomedicine to solid tumors. Nat. Rev. Clin. Oncol. 7, 653-664 (2010). https://doi. org/10.1038/nrclinonc.2010.139

49. H.C. Manning, N.B. Merchant, A.C. Foutch, J.M. Virostko, S.K. Wyatt et al., Molecular imaging of therapeutic response to epidermal growth factor receptor blockade in colorectal cancer. Clin. Cancer Res. 14, 7413-7422 (2008). https://doi. org/10.1158/1078-0432.CCR-08-0239

50. A.V. Salnikov, A. Groth, A. Apel, G. Kallifatidis, B.M. Beckermann et al., Targeting of cancer stem cell marker EpCAM by bispecific antibody EpCAMxCD3 inhibits pancreatic carcinoma. J. Cell Mol. Med. 13, 4023-4033 (2009). https://doi. org/10.1111/j.1582-4934.2009.00723.x

51. S. Liu, T.H. Bugge, S.H. Leppla, Targeting of tumor cells by cell surface urokinase plasminogen activator-dependent anthrax toxin. J. Biol. Chem. 276, 17976-17984 (2001). https ://doi.org/10.1074/jbc.M011085200

52. T. Lin, Q. Ren, W. Zuo, R. Jia, L. Xie et al., Valproic acid exhibits anti-tumor activity selectively against EGFR/ErbB2/ ErbB3-coexpressing pancreatic cancer via induction of ErbB family members-targeting microRNAs. J. Exp. Clin. Cancer Res. 38, 150 (2019). https://doi.org/10.1186/s1304 6-019-1160-9

53. O. Er, S.G. Colak, K. Ocakoglu, M. Ince, R. Bresoli-Obach et al., Selective photokilling of human pancreatic cancer cells using cetuximab-targeted mesoporous silica nanoparticles for delivery of zinc phthalocyanine. Molecules 23, 2749 (2018). https://doi.org/10.3390/molecules23112749

54. G. Obaid, S. Bano, S. Mallidi, M. Broekgaarden, J. Kuriakose et al., Impacting pancreatic cancer therapy in heterotypic in vitro organoids and in vivo tumors with specificitytuned, NIR-activable photoimmunonanoconjugates: towards conquering desmoplasia? Nano Lett. 19, 7573-7587 (2019). https://doi.org/10.1021/acs.nanolett.9b00859

55. B.Q. Spring, R. Bryan Sears, L.Z. Zheng, Z. Mai, R. Watanabe et al., A photoactivable multi-inhibitor nanoliposome for tumour control and simultaneous inhibition of treatment escape pathways. Nat. Nanotechnol. 11, 378-387 (2016). https ://doi.org/10.1038/nnano.2015.311

56. Y. Liu, P. Bhattarai, Z. Dai, X. Chen, Photothermal therapy and photoacoustic imaging via nanotheranostics in fighting cancer. Chem. Soc. Rev. 48, 2053-2108 (2019). https://doi. org/10.1039/c8cs00618k

57. R. Liu, L. Jing, D. Peng, Y. Li, J. Tian et al., Manganese (II) chelate functionalized copper sulfide nanoparticles for efficient magnetic resonance/photoacoustic dual-modal imaging guided photothermal therapy. Theranostics 5, 1144-1153 (2015). https://doi.org/10.7150/thno.11754

58. R. Liu, J. Tang, Y. Xu, Y. Zhou, Z. Dai, Nano-sized indocyanine green j-aggregate as a one-component theranostic agent. Nanotheranostics 1, 430-439 (2017). https://doi.org/10.7150/ ntno. 19935

59. J. Wang, X. Wu, P. Shen, J. Wang, Y. Shen et al., Applications of inorganic nanomaterials in photothermal therapy based on combinational cancer treatment. Int. J. Nanomed. 15, 19031914 (2020). https://doi.org/10.2147/IJN.S239751

60. A.H. Odda, H. Li, N. Kumar, N. Ullah, M.I. Khan et al., Polydopamine coated $\mathrm{PB}-\mathrm{MnO}_{2}$ nanoparticles as an oxygen generator nanosystem for imaging-guided single-NIR-laser triggered synergistic photodynamic/photothermal therapy. Bioconjug. Chem. 31, 1474-1485 (2020). https://doi. org/10.1021/acs.bioconjchem.0c00165

61. H.S. Jung, P. Verwilst, A. Sharma, J. Shin, J.L. Sessler et al., Organic molecule-based photothermal agents: an expanding photothermal therapy universe. Chem. Soc. Rev. 47, 22802297 (2018). https://doi.org/10.1039/c7cs00522a

62. W. Shao, C. Yang, F. Li, J. Wu, N. Wang et al., Molecular design of conjugated small molecule nanoparticles for synergistically enhanced PTT/PDT. Nano-Micro Lett. 12, 147 (2020). https://doi.org/10.1007/s40820-020-00474-6

63. M. Lan, S. Zhao, W. Liu, C.S. Lee, W. Zhang et al., Photosensitizers for photodynamic therapy. Adv. Healthc. Mater. 8, e1900132 (2019). https://doi.org/10.1002/adhm.201900132

64. S. Liu, X. Pan, H. Liu, Two-dimensional nanomaterials for photothermal therapy. Angew. Chem. Int. Ed. 59, 5890-5900 (2020). https://doi.org/10.1002/anie.201911477

65. Y. Li, Y. Wu, S. Zheng, X. Liang, X. Han et al., PEGylated cationic hybrid bicellar nanodisc for efficient siRNA delivery. RSC Adv. 6, 113745-113753 (2016). https://doi.org/10.1039/ c6ra24268e

66. W. Li, H. Zhang, X. Guo, Z. Wang, F. Kong et al., Gold nanospheres-stabilized indocyanine green as a synchronous photodynamic-photothermal therapy platform that inhibits tumor growth and metastasis. ACS Appl. Mater. Interfaces 9, 3354-3367 (2017). https://doi.org/10.1021/acsami.6b13351

67. H. He, S. Ji, Y. He, A. Zhu, Y. Zou et al., Photoconversiontunable fluorophore vesicles for wavelength-dependent 
photoinduced cancer therapy. Adv. Mater. 29, 1606690 (2017). https://doi.org/10.1002/adma.201606690

68. X. Li, Y. Liu, F. Fu, M. Cheng, Y. Liu et al., Single NIR laseractivated multifunctional nanoparticles for cascaded photothermal and oxygen-independent photodynamic therapy. Nano-Micro Lett. 11, 68 (2019). https://doi.org/10.1007/s4082 0-019-0298-5

69. W. Yin, T. Bao, X. Zhang, Q. Gao, J. Yu et al., Biodegradable MoOx nanoparticles with efficient near-infrared photothermal and photodynamic synergetic cancer therapy at the second biological window. Nanoscale 10, 1517-1531 (2018). https:// doi.org/10.1039/c7nr07927c

70. H. Li, P. Wang, Y. Deng, M. Zeng, Y. Tang et al., Combination of active targeting, enzyme-triggered release and fluorescent dye into gold nanoclusters for endomicroscopy-guided photothermal/photodynamic therapy to pancreatic ductal adenocarcinoma. Biomaterials 139, 30-38 (2017). https://doi. org/10.1016/j.biomaterials.2017.05.030

71. K. Ni, T. Aung, S. Li, N. Fatuzzo, X. Liang et al., Nanoscale metal-organic framework mediates radical therapy to enhance cancer immunotherapy. Chem 5, 1892-1913 (2019). https:// doi.org/10.1016/j.chempr.2019.05.013

72. A. Yuan, J. Wu, X. Tang, L. Zhao, F. Xu et al., Application of near-infrared dyes for tumor imaging, photothermal, and photodynamic therapies. J. Pharm. Sci. 102, 6-28 (2013). https:// doi.org/10.1002/jps.23356

73. Y. Liu, W. Zhen, L. Jin, S. Zhang, G. Sun et al., All-in-one theranostic nanoagent with enhanced reactive oxygen species generation and modulating tumor microenvironment ability for effective tumor eradication. ACS Nano 12, 4886-4893 (2018). https://doi.org/10.1021/acsnano.8b01893

74. X. Li, N. Kwon, T. Guo, Z. Liu, J. Yoon, Innovative strategies for hypoxic-tumor photodynamic therapy. Angew. Chem. Int. Ed. 57, 11522-11531 (2018). https://doi.org/10.1002/ anie. 201805138

75. H. Sun, Y. Zhang, S. Chen, R. Wang, Q. Chen et al., Photothermal fenton nanocatalysts for synergetic cancer therapy in the second near-infrared window. ACS Appl. Mater. Interfaces 12, 30145-30154 (2020). https://doi.org/10.1021/acsam i.0c07013

76. L. Yan, Y. Wang, T. Hu, X. Mei, X. Zhao et al., Layered double hydroxide nanosheets: towards ultrasensitive tumor microenvironment responsive synergistic therapy. J. Mater. Chem. B 8, 1445-1455 (2020). https://doi.org/10.1039/c9tb02591j

77. Y. Han, S. Gao, Y. Zhang, Q. Ni, Z. Li et al., Metal-based nanocatalyst for combined cancer therapeutics. Bioconjug. Chem. 31, 1247-1258 (2020). https://doi.org/10.1021/acs. bioconjchem.0c00194

78. P. Wang, C. Liang, J. Zhu, N. Yang, A. Jiao et al., Manganesebased nanoplatform as metal ion-enhanced ROS generator for combined chemodynamic/photodynamic therapy. ACS Appl. Mater. Interfaces 11, 41140-41147 (2019). https://doi. org/10.1021/acsami.9b16617

79. Z. Huang, H. Moseley, S. Bown, Rationale of combined PDT and SDT modalities for treating cancer patients in terminal stage: the proper use of photosensitizer. Integr. Cancer Ther.
9, 317-319 (2010). https://doi.org/10.1177/153473541037663 4

80. J.N. Kenyon, Outcome measures following sonodynamic photodynamic therapy: a case series. Curr. Drug. Ther. 6, 12-16 (2011). https://doi.org/10.2174/157488511794079059

81. Y. Zhou, M. Wang, Z. Dai, The molecular design of and challenges relating to sensitizers for cancer sonodynamic therapy. Mater. Chem. Front. 4, 2223-2234 (2020). https://doi. org/10.1039/d0qm00232a

82. C. McEwan, H. Nesbitt, D. Nicholas, O.N. Kavanagh, K. McKenna et al., Comparing the efficacy of photodynamic and sonodynamic therapy in non-melanoma and melanoma skin cancer. Bioorg. Med. Chem. 24, 3023-3028 (2016). https:// doi.org/10.1016/j.bmc.2016.05.015

83. G.Y. Wan, Y. Liu, B.W. Chen, Y.Y. Liu, Y.S. Wang et al., Recent advances of sonodynamic therapy in cancer treatment. Cancer Biol. Med. 13, 325-338 (2016). https://doi. org/10.20892/j.issn.2095-3941.2016.0068

84. S. Binder, B. Hosikova, Z. Mala, L. Zarska, H. Kolarova, Effect of ClAlPcS(2) photodynamic and sonodynamic therapy on HeLa cells. Physiol. Res. 68, S467-S474 (2019). https:// doi.org/10.33549/physiolres.934374

85. M. Bakhshizadeh, T. Moshirian, H. Esmaily, O. Rajabi, H. Nassirli et al., Sonophotodynamic therapy mediated by liposomal zinc phthalocyanine in a colon carcinoma tumor model: role of irradiating arrangement. Iran. J. Basic Med. Sci. 20, 1088-1092 (2017). https://doi.org/10.22038/IJBMS .2017 .9410

86. P. Wang, C. Li, X. Wang, W. Xiong, X. Feng et al., Anti-metastatic and pro-apoptotic effects elicited by combination photodynamic therapy with sonodynamic therapy on breast cancer both in vitro and in vivo. Ultrason. Sonochem. 23, 116-127 (2015). https://doi.org/10.1016/j.ultsonch.2014.10.027

87. Y. Liu, P. Wang, Q. Liu, X. Wang, Sinoporphyrin sodium triggered sono-photodynamic effects on breast cancer both in vitro and in vivo. Ultrason. Sonochem. 31, 437-448 (2016). https:// doi.org/10.1016/j.ultsonch.2016.01.038

88. J. Chen, H. Luo, Y. Liu, W. Zhang, H. Li et al., Oxygen-selfproduced nanoplatform for relieving hypoxia and breaking resistance to sonodynamic treatment of pancreatic cancer. ACS Nano 11, 12849-12862 (2017). https://doi.org/10.1021/ acsnano.7b08225

89. K. Wang, Y. Zhang, J. Wang, A. Yuan, M. Sun et al., Selfassembled IR780-loaded transferrin nanoparticles as an imaging, targeting and PDT/PTT agent for cancer therapy. Sci. Rep. 6, 27421 (2016). https://doi.org/10.1038/srep27421

90. C. Hu, L. Cai, S. Liu, Y. Liu, Y. Zhou et al., Copper-doped nanoscale covalent organic polymer for augmented photo/ chemodynamic synergistic therapy and immunotherapy. Bioconjug. Chem. 31, 1661-1670 (2020). https://doi.org/10.1021/ acs.bioconjchem.0c00209

91. C. Li, J. Wang, Y. Wang, H. Gao, G. Wei et al., Recent progress in drug delivery. Acta Pharm. Sin. B 9, 1145-1162 (2019). https://doi.org/10.1016/j.apsb.2019.08.003

92. F. Zhang, Q. Wu, H. Liu, NIR light-triggered nanomaterials-based prodrug activation towards cancer therapy. Wiley 
Interdiscip. Rev. Nanomed. Nanobiotechnol. 12, e1643 (2020). https://doi.org/10.1002/wnan.1643

93. Y. Zhang, F. Huang, C. Ren, L. Yang, J. Liu et al., Targeted chemo-photodynamic combination platform based on the DOX prodrug nanoparticles for enhanced cancer therapy. ACS Appl. Mater. Interfaces 9, 13016-13028 (2017). https://doi. org/10.1021/acsami.7b00927

94. T. Zhang, Z. Jiang, L. Chen, C. Pan, S. Sun et al., PCN-Fe(III)PTX nanoparticles for MRI guided high efficiency chemophotodynamic therapy in pancreatic cancer through alleviating tumor hypoxia. Nano Res. 13, 273-281 (2020). https://doi. org/10.1007/s12274-019-2610-6
95. S. Liang, X. Deng, Y. Chang, C. Sun, S. Shao et al., Intelligent hollow Pt-CuS janus architecture for synergistic catalysis-enhanced sonodynamic and photothermal cancer therapy. Nano Lett. 19, 4134-4145 (2019). https://doi.org/10.1021/acs. nanolett.9b01595

96. V.S. Madamsetty, K. Pal, S.K. Dutta, E. Wang, J.R. Thompson et al., Design and evaluation of PEGylated liposomal formulation of a novel multikinase inhibitor for enhanced chemosensitivity and inhibition of metastatic pancreatic ductal adenocarcinoma. Bioconjug. Chem. 30, 2703-2713 (2019). https:// doi.org/10.1021/acs.bioconjchem.9b00632 